\title{
Connaissances en radioprotection des travailleurs exposés aux rayonnements ionisants en milieu médical en Afrique francophone sub-saharienne
}

\author{
D.W.M.M. Akanni ${ }^{1, a,}{ }^{*}$, K.-M. Savi de Tové $^{1}$, B.G. Damien ${ }^{2}$, S.M. Kiki ${ }^{1}$, S.B.M.G. Adjadohoun ${ }^{3}$, \\ P. Yekpe-Ahouansou ${ }^{3}$ et K.P. N'zi ${ }^{4}$ \\ ${ }^{1}$ Faculté de médecine, Université de Parakou, Parakou, Bénin. \\ 2 Centre de formation et de recherche en matière de population, Université d'Abomey-Calavi, Abomey-Calavi, Bénin. \\ ${ }^{3}$ Faculté des sciences de la santé, Université d'Abomey-Calavi, Abomey-Calavi, Bénin. \\ ${ }^{4}$ Institut de cardiologie d'Abidjan, Université Félix Houphouët Boigny, Abidjan, Côte-d'Ivoire.
}

Reçu le 25 avril 2020 / Accepté le 24 août 2020

\begin{abstract}
Résumé - L'objectif de cette étude était de mesurer le niveau de connaissances en radioprotection des travailleurs exposés aux rayonnements ionisants en milieu médical en Afrique francophone sub-saharienne. Une étude transversale sous forme de questionnaire a été réalisée à Cotonou en mai 2019 durant les Journées de radiologie d'Afrique noire francophone (JRANF). Les connaissances ont été évaluées sur les sources des rayonnements ionisants $(\mathrm{RI})$, les principes élémentaires de la radioprotection, les effets biologiques des $\mathrm{RI}$, les effets pathologiques des RI, les grandeurs et unités en radioprotection, la radioprotection des patients, la grossesse et l'exposition médicale aux RI. Le score global des connaissances (SGC) a été calculé à partir du nombre de bonnes réponses aux questions $(n=20)$ et classé en «Bon», «Moyen» ou «Mauvais». La relation entre la qualification professionnelle des travailleurs et leurs connaissances en radioprotection a été analysée. La population d'étude était composée de 91 travailleurs. La moyenne du SGC était de 7,9 avec des valeurs extrêmes de 2 et 12. Le SGC était classé «Mauvais » chez 93,41\% de la population d'étude et aucun des enquêtés n'avait un SCG classé «Bon». Le SGC des manipulateurs était deux fois plus petit que celui des DES-RIM $(p<0,0001)$ et trois fois plus petit que celui des médecins radiologues $(p<0,0001)$.
\end{abstract}

Mots clés : radioprotection / connaissances / Afrique / formation continue

\begin{abstract}
Radiation protection knowledge of workers exposed to ionizing radiation in medical practice in French-speaking Sub-Saharan Africa. The study aimed to assess the level of knowledge in radiation protection of workers exposed to ionizing radiation in medical settings in French-speaking SubSaharan Africa. A cross-sectional survey was carried out at Cotonou in Benin country in May 2019 during the French-speaking Africa radiology meeting (JRANF). Knowledge was evaluated regarding the source of ionizing radiation (IR), the basic principles of radiation protection, the biological effects of IR, the pathological effects of IR, the quantities and units in radiation protection, the radiation protection of patients, pregnancy medical exposure to IR. The Overall Knowledge Score (OKS) was calculated based on the number of correct answers to the 20 questions and classified as "Good", "Average" or "Poor". The links between the worker's professional qualifications and their knowledge in radiation protection was tested. A total of 91 workers were included in the study. The average OKS was 7.9 with extreme values of 2 and 12. The OKS was poor in $93.41 \%$ of the survey population and none of the respondents had a good OKS. The OKS of the technicians was two times lower than resident's $(P<0.0001)$ and three times lower than radiologist's $(P<0.0001)$.
\end{abstract}

Keywords: radiation protection / knowledge / Africa / continuing education

\footnotetext{
*Auteur de correspondance : djivakanni@yahoo.fr

${ }^{a}$ Adresse personnelle: 06 BP 3263, Cotonou, Bénin.
} 


\section{Introduction}

La radioprotection désigne la protection des personnes contre les effets d'une exposition à des rayonnements ionisants (RI) et les moyens d'assurer cette protection (AIEA, 2007). L'utilisation des RI à des fins médicales, principale source artificielle d'exposition (AIEA, 2014), constitue un véritable dilemme. Depuis plusieurs décennies, l'Agence internationale de l'énergie atomique (AIEA) et la Commission internationale de protection radiologique (CIPR) publient régulièrement des recommandations invitant les États membres à un usage contrôlé et sécurisé des RI à des fins médicales, à travers deux grands principes : la justification et l'optimisation (N'zi et al., 2016). L'AIEA recommande que les connaissances des travailleurs directement affectés aux travaux sous rayonnements ionisants (DATR) sur les fondements de la radioprotection et de la sûreté, leur niveau de formation, leurs aptitudes et compétences à effectuer sans risque les tâches spécifiées soient évaluées et confirmées adéquates, avant toute affectation non supervisée à un poste de travail (AIEA, 2001). La radioprotection est, de ce fait, une obligation.

En Europe, les États membres de l'AIEA veillent à ce que des mesures soient prises pour organiser l'enseignement, la formation et le recyclage de manière à permettre la reconnaissance des experts en radioprotection et des experts en physique médicale, ainsi que des services de médecine du travail et des services de dosimétrie, en relation avec le type de pratiques (EURATOM, 2014).

Dans plusieurs pays africains, l'encadrement de la radioprotection demeure, à nos jours, insuffisant en raison entre autres du manque d'information, de l'absence de formation professionnelle appropriée, de l'inexistence et/ou de la faiblesse des services d'inspection de radioprotection (Ongolo-Zogo et al., 2013). Cette situation se retrouve également dans d'autres pays, même si la formation continue y est plus développée (Aldhafeeri, 2020). Au vu de ces constats, l'AIEA a initié plusieurs ateliers de formation au profit des utilisateurs des RI (radiologues, spécialiste de médecine nucléaire, techniciens, physiciens médicaux), des managers (ex. national liaison officier [NLO]), et des autorités de régulation. Mais l'impact de ces formations sur l'ensemble des acteurs du système reste limité (N'zi et al., 2016).

La Société des radiologistes d'Afrique noire francophone (SRANF) organise depuis 2018 avec l'aide de ses partenaires notamment l'AIEA, le Collège des enseignants en radiologie de France (CERF) et la Société française de radiologie (SFR) une formation post-universitaire en radioprotection destinée aux utilisateurs des RI pendant les Journées de radiologie d'Afrique noire francophone (JRANF). Les $15^{\text {es }}$ JRANF tenues à Cotonou en mai 2019 nous ont permis de mener cette étude qui avait pour principal objectif d'évaluer les connaissances en radioprotection des travailleurs exposés aux rayonnements ionisants en milieu médical en Afrique francophone sub-saharienne.

\section{Cadre et méthodes d'étude}

Au cours des $15^{\mathrm{e}}$ JRANF tenues à Cotonou au Bénin du 22 au 24 mai 2019, une formation post-universitaire en radioprotection a été organisée. La formation a duré dix heures réparties en deux demi-journées. Les participants étaient les travailleurs directement affectés aux travaux sous rayonnement (DATR) en milieu médical dans les pays africains francophones sub-sahariens. Il s'agissait des médecins radiologues, des médecins en spécialisation inscrits aux diplômes d'études spéciales en radiologie et imagerie médicale (DES-RIM), des manipulateurs en électroradiologie, des physiciens médicaux et des médecins nucléaires. L'enseignement dispensé au cours de cette formation post-universitaire portait sur :

- les unités de dose en radioprotection;

- les risques déterministes et stochastiques;

- la mesure pratique des niveaux d'exposition en radiologie conventionnelle et en tomodensitométrie ;

- les niveaux comparés des doses observées en radiologie diagnostique et interventionnelle;

- la justification des actes et l'optimisation;

- les méthodes d'optimisation en radiologie conventionnelle;

- les méthodes d'optimisation en tomodensitométrie;

- la grossesse et l'exposition médicale;

- le cas particulier de l'enfant et de l'exposition aux RI;

- la justification des actes et les guidelines.

À la fin de la formation, une étude transversale descriptive analytique basée sur le post-test de la formation a été menée auprès des participants. Nous n'avons retenu que les participants qui ont suivi la formation en radioprotection et qui ont rempli le questionnaire du post-test.

Le questionnaire du post-test était semi-structuré et élaboré selon les objectifs généraux et spécifiques de la formation nationale en radioprotection destinée aux internes de radiologie de France. Il comportait 20 questions regroupées en sept parties évaluant (Tab. S1) :

- les caractéristiques socioprofessionnelles : sexe, qualification, pays de provenance;

- les connaissances sur les sources d'irradiation (naturelles et artificielles) et les principes élémentaires de radioprotection (justification, optimisation, limitation des doses);

- les connaissances sur les effets biologiques des radiations ionisantes (mort cellulaire, lésions d'ADN, radiosensibilité des lignées cellulaires jeunes), et sur le lien entre la relation linéaire sans seuil et le risque cancérogène;

- les connaissances sur les risques stochastiques et déterministes, les risques tératogènes des radiations ionisantes ;

- les connaissances sur les grandeurs et unités de mesure de dose en radiologie conventionnelle et en tomodensitométrie ;

- les connaissances sur la radioprotection des patients : niveau de référence des procédures de diagnostic, guide de procédures et indications des examens;

- les connaissances sur l'exposition médicale aux rayonnements ionisants pendant la grossesse.

Chaque bonne réponse était cotée d'une valeur de 1 et chaque mauvaise réponse cotée d'une valeur de 0 . Le score global des connaissances (SGC) a été comptabilisé à partir du nombre de bonnes réponses aux questions $(n=20)$. Le niveau des connaissances était classé «Bon» si le SGC était supérieur à 16 , «Moyen» si le SGC était compris entre 12 et 16 , et «Mauvais» lorsque le SGC est inférieur à 12 (Marzouk Moussa et Kamoun, 2016). 
Tableau 1. Répartition des travailleurs directement affectés aux travaux sous rayonnement ayant participé à l'étude en fonction du sexe, de la qualification professionnelle et du pays d'exercice.

Table 1. Distribution of workers directly assigned to radiation work participating to the study according to gender, professional qualification and country of practice.

\begin{tabular}{|c|c|c|}
\hline & Effectif & Proportion $(\%)$ \\
\hline \multicolumn{3}{|l|}{ Sexe } \\
\hline Féminin & 56 & 61,54 \\
\hline Masculin & 35 & 38,46 \\
\hline \multicolumn{3}{|c|}{ Qualification professionnelle } \\
\hline DES-RIM & 43 & 47,25 \\
\hline Manipulateurs & 35 & 38,46 \\
\hline Radiologues & 13 & 14,29 \\
\hline \multicolumn{3}{|l|}{ Pays d'exercice } \\
\hline Bénin & 44 & 48,35 \\
\hline Togo & 33 & 36,26 \\
\hline Burkina Faso & 3 & 03,29 \\
\hline Niger & 3 & 03,29 \\
\hline Gabon & 3 & 03,29 \\
\hline Autres ${ }^{*}$ & 5 & 05,49 \\
\hline
\end{tabular}

${ }^{*}$ Cameroun, Congo, Guinée, Tchad, Sénégal.

Pour l'analyse statistique, nous avons utilisé le logiciel STATA, version 12 (Stata Corp., TX, États-Unis). Nous avons calculé les fréquences pour les variables qualitatives, les moyennes et les écarts-types pour les variables quantitatives. Ensuite, nous avons testé l'association entre la qualification professionnelle regroupée en trois modalités (médecins radiologues, DES-RIM et manipulateurs) et le SGC. Pour cela, la comparaison de la distribution du SGC entre les trois catégories de qualification professionnelle a été réalisée grâce au test statistique ANOVA. Une différence entre les moyennes a été considérée comme statistiquement significative si $p$-value est inférieur à 0,05 .

\section{Résultats}

\subsection{Les caractéristiques socioprofessionnelles de la population d'étude}

Notre échantillon était composé de 91 travailleurs. La majorité de la population d'étude était de sexe féminin $(61,54 \%)$. La sex-ratio $(\mathrm{H} / \mathrm{F})$ était de 0,62 . Les participants à notre étude provenaient de dix pays francophones subsahariens. Les pays les plus représentés étaient le Bénin $(48,35 \%)$ et le Togo $(36,26 \%)$. Ils étaient $43(47,25 \%)$

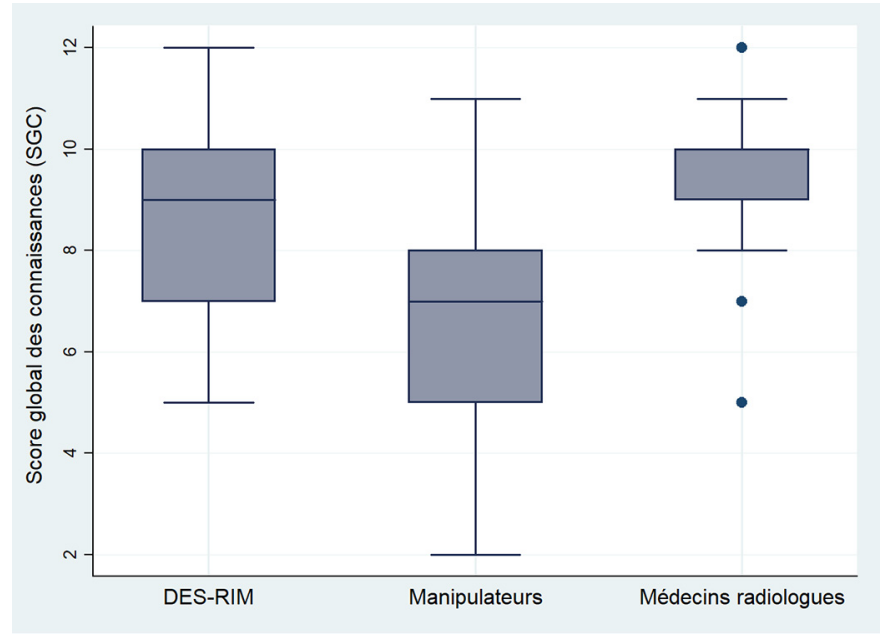

Fig. 1. Score global des connaissances en fonction de la qualification professionnelle des travailleurs directement affectés aux travaux sous rayonnement en Afrique francophone sub-saharienne. Les trois boîtes à moustaches représentent chacune la distribution du SGC des DESRIM, des manipulateurs et des médecins radiologues. Les barres horizontales indiquent la médiane du SGC au niveau de chaque boîte. Les bords inférieurs et supérieurs de chaque boîte indiquent les intervalles interquartiles. Les moustaches supérieures indiquent les valeurs maximales et les moustaches inférieures indiquent les valeurs minimales de la distribution du SGC. Il y avait une différence statistiquement significative entre le SGC des manipulateurs et celui des DES-RIM $(p<0,0001)$ et entre le SGC des médecins radiologues $(p<0,0001)$. Il n'y avait pas de différence entre le SGC des DESRIM et celui des médecins radiologues.

Fig. 1. Overall Knowledge Score according to the qualification of French-speaking Sub-Saharan African workers directly assigned to radiation work.

médecins inscrits au DES-RIM, 35 (38,46\%) manipulateurs et $13(14,29 \%)$ médecins radiologues. Le tableau 1 montre la répartition des DATR en fonction du sexe, de leur pays de provenance et de leur qualification professionnelle.

\subsection{Répartition de la population étudiée selon le score globale de connaissance}

La moyenne du SGC de la population d'étude était de $7,9 \pm 2,3$ avec des valeurs extrêmes de 2 et 12 . Aucun des enquêtés n'avait un SGC classé «Bon». Un SGC «Moyen» a été observé chez $6,59 \%$ des participants et un SGC «Mauvais » chez 93,41\% d'entre eux soit 85 participants.

La moyenne la plus élevée du SGC a été observée chez les médecins radiologues $(9,4 \pm 1,9)$ avec des valeurs extrêmes de 5 et 12. La moyenne du SGC des DES-RIM était de $8,6 \pm 1,9$ avec des valeurs extrêmes de 5 et 12 . La moyenne la plus basse du SGC a été observée chez les manipulateurs $(6,7 \pm 2,2)$ avec des valeurs extrêmes de 2 et 11 . La figure 1 présente la distribution des SGC en fonction de la qualification professionnelle. Grâce au test ANOVA, la distribution du SGC a été comparée en fonction des qualifications professionnelles. Le SGC des DES-RIM était 1,89 fois plus élevée que celui des manipulateurs $(p<0,0001)$. De même, le SGC 
des radiologues était 2,69 fois plus élevée que celui des manipulateurs $(p<0,0001$,$) . Il n'existait pas de différence$ statistiquement significative entre le SGC des DES-RIM et celui des médecins radiologues $(p=0,2190)$.

\subsection{Analyse des niveaux de connaissance par thème}

3.3.1 Connaissances sur les principes élémentaires de la radioprotection et les sources des rayonnements ionisants

La connaissance des principes élémentaires de la radioprotection était bonne chez $86,81 \%(n=79)$ des participants. Plus précisément, la connaissance de la justification, de l'optimisation et de la limitation des doses était bonne respectivement chez $100 \%$ des médecins radiologues, $88,37 \%$ des DES-RIM et $80 \%$ des manipulateurs. La connaissance des sources des RI était bonne chez 36,26\% $(n=33)$ des travailleurs exposés. La bonne connaissance à la fois des sources de RI et des principes élémentaires de la radioprotection était faible et retrouvée chez $34,07 \%(n=31)$ des participants.

\subsubsection{Connaissances sur les effets biologiques des rayonnements ionisants}

La connaissance des effets biologiques des RI était mauvaise chez $74,73 \%(n=68)$ des participants et $82,42 \%$ $(n=75)$ d'entre eux avaient une mauvaise connaissance du lien entre «la relation linéaire sans seuil» et le risque cancérigène des RI.

La bonne connaissance du lien entre «la relation linéaire sans seuil » et le risque cancérigène des RI était retrouvée chez seulement $4,40 \%(n=4)$ des participants.

\subsubsection{Connaissances sur les effets pathologiques des rayonnements ionisants}

La connaissance sur les risques déterministes était mauvaise chez $63,74 \%(n=58)$ des participants et $85,71 \%$ $(n=78)$ d'entre eux avaient également une mauvaise connaissance sur les risques stochastiques. La connaissance sur les risques tératogènes des RI était mauvaise chez $61,54 \%$ $(n=56)$ des participants. En outre, 86,81\% $(n=79)$ ne connaissaient pas les effets pathologiques des RI sur l'organisme des enfants.

Un seul participant à l'étude avait une bonne connaissance à la fois des risques déterministes, stochastiques, tératogènes et des effets pathologiques des RI sur l'organisme des enfants.

\subsubsection{Connaissances sur les grandeurs et unités de mesure de dose en radiologie conventionnelle et en tomodensitométrie}

Une bonne connaissance de l'unité de mesure de la dose délivrée en radiologie conventionnelle était retrouvée chez $92,31 \%(n=84)$ des travailleurs exposés.

Par contre, il y avait une mauvaise connaissance respectivement :

- de l'unité de mesure de la dose efficace chez 71,43\% $(n=65)$;

- de l'unité de mesure de la dose délivrée en tomodensitométrie chez $60,44 \%(n=55)$;
- de l'unité de mesure de la dose absorbée par les tissus chez $47,25 \%(n=43)$ des participants.

Huit sujets, soit $8,79 \%$ des travailleurs exposés, avaient une bonne connaissance à la fois des éléments caractérisant la dose efficace, de l'unité de mesure de la dose délivrée respectivement en tomodensitométrie et en radiologie conventionnelle; et de l'unité de mesure de la dose absorbée par les tissus.

\subsubsection{Connaissances sur la radioprotection des patients}

Une bonne connaissance des méthodes d'optimisation des doses chez l'enfant était notée chez $87,91 \%(n=80)$ des participants.

Le niveau de connaissance était mauvais sur les méthodes d'optimisation des doses en radiologie conventionnelle chez $77,78 \%(n=70)$ des participants et en tomodensitométrie chez $71,43 \%(n=65)$ des participants, respectivement.

Il y avait une mauvaise connaissance de la dose délivrée en radiologie conventionnelle chez $67,03 \%(n=61)$ des participants.

Le niveau de la dose délivrée par les radiographies et les tomodensitométries était méconnu respectivement par 94,51\% $(n=86)$ des participants chez l'enfant et par 93,41\% $(n=85)$ des participants chez l'adulte.

Un seul participant avait une bonne connaissance à la fois :

- des méthodes d'optimisation des doses chez l'enfant;

- des méthodes d'optimisation des doses en radiologie conventionnelle et en tomodensitométrie;

- du niveau de la dose délivrée en radiologie conventionnelle et en tomodensitométrie aussi bien chez l'enfant que chez l'adulte.

\subsubsection{Connaissance sur la grossesse et l'exposition médicale aux rayonnements ionisants}

Une mauvaise connaissance des risques radiques chez la femme enceinte était observée chez $28,57 \%(n=26)$ des enquêtés. Ils étaient respectivement quatre radiologues, neuf DES RIM et treize manipulateurs.

\section{Discussion}

\subsection{Forces et limites de l'étude}

Notre étude est la toute première qui a évalué les connaissances en radioprotection chez les travailleurs exposés aux RI en milieu médical de dix pays francophones subsahariens, membres de la SRANF. Le questionnaire utilisé était basé sur les objectifs d'apprentissage généraux et spécifiques en radioprotection des internes inscrits au DES de Radiologie en France, qui ne reflète pas forcément les spécificités africaines notamment en matière du niveau d'irradiation des examens de radiodiagnostic et les sources de RI. Le fait que cette étude ait été réalisée sous forme d'un post-test d'une formation post-universitaire lors des JRANF a contribué également à limiter le nombre de participants à l'étude, mais la diversité de leur origine géographique constitue un atout. 


\subsection{Score global de connaissance}

Notre étude a montré que $93,41 \%$ des DATR avaient un SCG classé «Mauvais». Le SCG était classé «Mauvais» chez les trois groupes professionnels (médecins radiologues, DES-RIM, manipulateurs) et notamment aucun enquêté des trois groupes n'a eu un SGC classé «Bon ». Il n'y avait pas de différence statistiquement significative entre les médecins radiologues et les DES-RIM en formation initiale. Mais il a été retrouvé une différence significative entre le SGC des DESRIM et celui des manipulateurs, d'une part, et entre le SGC des radiologues et celui des manipulateurs, d'autre part $(p<0,0001)$. En effet, malgré la faiblesse de leur niveau de connaissance en radioprotection les médecins (radiologues et DES-RIM) avaient une meilleure connaissance comparée aux manipulateurs. Cette différence avait également été observée au Cameroun (Ongolo-Zogo et al., 2013) et en Tunisie (Marzouk Moussa et Kamoun, 2016).

Le faible niveau de connaissance globale de la radioprotection des travailleurs exposés avait déjà été observé de façon isolée dans les différents pays membres de la SRANF notamment au Cameroun (Moifo et al., 2017), au Burkina Faso (Nikiema et al., 2017), et en Centrafrique (Kouandongui Bangué Songrou et al., 2019). Cette mauvaise connaissance globale de la radioprotection par les travailleurs exposés n'était pas spécifique aux pays francophones sub-sahariens. Une étude quasi similaire réalisée en 2015 en Tunisie a montré que $59 \%$ des travailleurs exposés avaient un SGC classé «Mauvais » (Marzouk Moussa et Kamoun, 2016). La moyenne du SCG des travailleurs exposés dans cette étude était de 11,8 avec des valeurs limites de 1 et 18 (Marzouk Moussa et Kamoun, 2016). Cette moyenne quoique légèrement plus élevée que la nôtre demeure faible. Une autre étude en Arabie Saoudite a également montré un déficit de connaissances en radioprotection des DATR (Aldhafeeri, 2020).

\subsection{Analyse des niveaux de connaissance par thème}

Les travailleurs exposés au RI ionisant dans notre étude avaient une connaissance jugée mauvaise sur :

- les risques tératogènes des RI;

- les risques stochastiques;

- les risques déterministes;

- les grandeurs et unités de dose en radioprotection;

- les méthodes d'optimisation de dose en radiologie conventionnelle et en tomodensitométrie ;

- les niveaux de doses délivrées en radiologie conventionnelle et tomodensitométrie.

Seuls les principes élémentaires de la radioprotection étaient bien connus par les travailleurs exposés au RI en milieu médical africain francophone sub-saharien.

En effet, dans les pays francophones sub-sahariens, l'absence de formateur qualifié fait que la formation initiale et la formation continue sont inexistantes (Mbo Amvene et al., 2017; Guiegui et al., 2019).

Malgré le nombre croissant des installations radiologiques et notamment d'installations d'imagerie en coupe (tomodensitométrie) en Afrique sub-saharienne, il n'existe pas souvent de centre de dosimétrie (Tapsoba et al., 2012). L'absence de la dosimétrie a pour corollaire l'absence de niveaux de référence diagnostiques pour les examens radiologiques les plus couramment pratiqués. Il n'existe pas non plus de guide de procédures de réalisation des examens d'imagerie (N'zi et al., 2016).

Pour être efficace, la mise en œuvre de la radioprotection s'appuie sur une institution administrative locale indépendante (autorité de radioprotection), qui sensibilise la population sur les dangers et les risques liés à l'utilisation des RI et contribue également à la formation des professionnels de la santé sur la radioprotection (CIPR, 2007; Aubert, 2010). Dans la majorité des pays de la SRANF, cette institution locale chargée de la radioprotection n'existe pas ou n'est pas fonctionnelle. Lorsqu'elle existe, elle n'émet pas de directives nationales (N'zi et al., 2016).

L'absence de législation au niveau national avec comme conséquence le défaut de formation initiale et/ou continue et l'implication insuffisante sur le terrain des autorités de radioprotection dans les différents pays de l'Afrique francophone sub-saharienne peuvent expliquer le SGC classé «Mauvais» des travailleurs exposés.

\section{Conclusion}

La connaissance en radioprotection des travailleurs exposés en milieu médical dans les pays africains francophones sub-sahariens est globalement mauvaise. Seuls, les principes élémentaires de la radioprotection sont connus. Les médecins (radiologues et DES-RIM) avaient un niveau de connaissance en radioprotection supérieur à celui des manipulateurs. La mise en place par la SRANF d'un programme de formation initiale et continue en radioprotection commune à tous les pays membres ainsi que des guides de procédures de réalisation des examens d'imagerie pourrait permettre de relever le niveau de connaissances des travailleurs exposés.

\section{Matériel supplémentaire}

Tableau S1. Questions - Examen de radioprotectionCotonou, mai 2019.

Le matériel supplémentaire est disponible sur https://www. radioprotection.org//10.1051/radiopro/2020067/olm.

\section{Références}

AIEA. 2001. Radiological protection for medical exposure to ionizing radiation. Safety standards series No. RS-G-1.5. Vienna: IAEA.

AIEA. 2007. Glossaire de sûreté de l'AIEA - Terminologie employée en sûreté nucléaire et radioprotection. Vienna: IAEA.

AIEA. 2014. Bulletin 55. Vienna: IAEA.

Aldhafeeri FM. 2020. Radiographers' knowledge regarding patients' ionizing radiation doses during common radiological procedures in Saudi Arabia. Radioprotection 55(1): 55-60.

Aubert B. 2010. Organisation de la radioprotection en France. J. Radiol. 91: 1201-1206.

CIPR. 2007. Recommandations CIPR 2007, publication 103. Paris: Éditions TEC\&DOC, 2009: 415.

EURATOM. 2014. Directive 2013/59/EURATOM du Conseil du 5 décembre 2013. Journal officiel de l'Union européenne du 17/01/ 2014. 
Guiegui CP, Manga Owona LJ, Aka INA, N'gassam Tchouasseu L, Afoue N'guessan LM, Kra AAC, Ya Ouattara M, Wognin Sangah B. 2019. Observation des mesures de radioprotection en milieu hospitalier à Yaoundé en 2016. Health Sci. Dis. 20(1): 91-94.

Kouandongui Bangué Songrou F, Bidan Tapiade E, Ouimon M, Mobima T. 2019. Connaissances en matière de radioprotection des manipulateurs de radiologie de Bangui et de Bimbo (Centrafrique). Radioprotection 54(1): 41-45

Marzouk Moussa I, Kamoun H. 2016. Connaissances des travailleurs en radioprotection - Enquête au CHU Mongi Slim à La Marsa (Tunisie). Radioprotection 51(2): 123-128.

Mbo Amvene J, Djonyang B, Mballa Amougou JC, Ngaroua D, Nko'o Amvene S. 2017. Observance des règles de radioprotection dans les services d'imagerie de l'Extrême-Nord du Cameroun. Health Sci. Dis. 18(2): 83-87.

Moifo B, Tene U, Moulion Tapouh JR, Samba Ngano O, Tchemtchoua Youta J, Simo A, Gonsu Fotsin J. 2017. Knowledge on irradiation, medical imaging prescriptions, and clinical imaging referral guidelines among physicians in sub-Saharan African country (Cameroun). Hindawi Radiol. Res. Pract. 2017: 1245236, 7 p., https://doi.org/10.1155/2017/1245236.

Nikiema Z, Tankoano AI, Bicaba D, Sombie I, Zoungrana B, Cisse R. 2017. Évaluation des connaissances des praticiens sur la radioprotection des patients au Burkina Faso. J. Afr. Imag. Med. 9(3): 101-105.

N'zi KP, Ahoury NJ, Comoé CP, Seka AR. 2016. Radioprotection des patients en Afrique sub-saharienne francophone: état des lieux, perspectives. J. Afr. Imag. Med. 8(2): 52.

Ongolo-Zogo P, Nguehouo MB, Yomi J, Nko'o Amven S. 2013. Connaissances en matière de radioprotection: enquête auprès des personnels des services hospitaliers de radiodiagnostic, radiothérapie et médecine nucléaire à Yaoundé Cameroun. Radioprotection 48(1): 39-49.

Tapsoba TL, Ouattara F, Ouédraogo S, Sanon H, Yanaba DH, Gansonré V. 2012. État du suivi dosimétrique des travailleurs dans les services de radiologie de la ville de Ouagadougou. $\mathrm{Med}$. Nucl. 36(2012): 539-544

Citation de l'article : Akanni DWMM, Savi de Tové K-M, Damien BG, Kiki SM, Adjadohoun SBMG, Yekpe-Ahouansou P, N'zi KP. 2021. Connaissances en radioprotection des travailleurs exposés aux rayonnements ionisants en milieu médical en Afrique francophone sub-saharienne. Radioprotection 56(1): 43-48 